\title{
La Guía de Trabajo Autónomo como herramienta imprescindible en el modelo de EA "Vaula"
}

Huertas, Ana ${ }^{a}$, Molina, Francisca ${ }^{\mathrm{b}}$ y Rosales, Consuelo ${ }^{\mathrm{c}}$

âniversidad de Jaén, mhuertas@ujaen.es, bUniversidad de Jaén, mfmolina@ujaen.es y 'Cuniversidad de Jaén, mrosales@ujaen.es.

\section{\$EWBDFW}

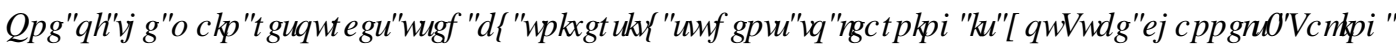

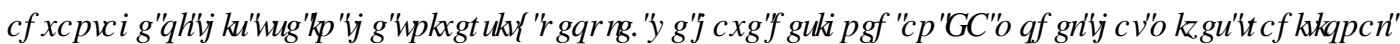

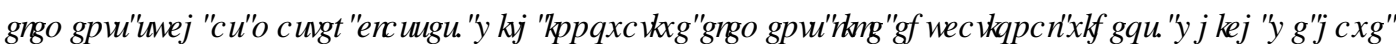

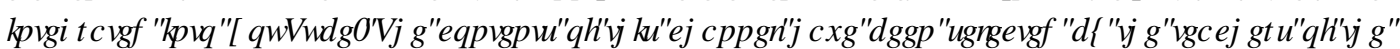

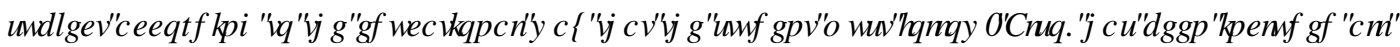
WKRUHFRQWQWUWXDKDYHEHQSSLRSVHGEI IUKHWXGHQW

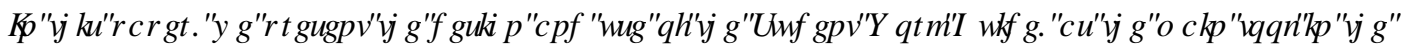

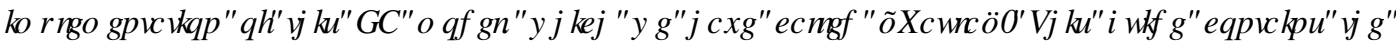

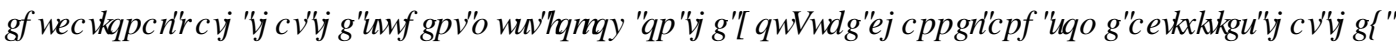
P XWU RLNRXWURIP SLRYHIKHUUNH IFRP SHMQFIHWD

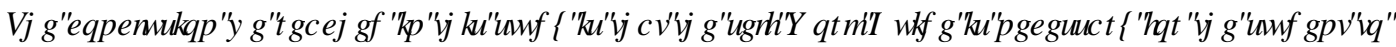

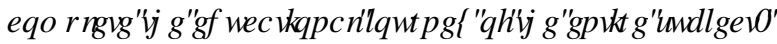

(

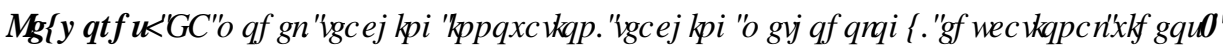
]

\section{HXP HQ}

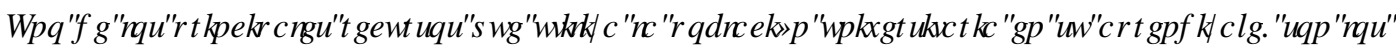

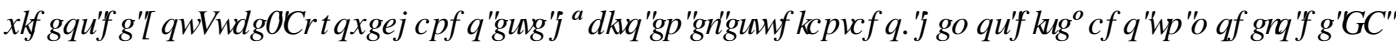

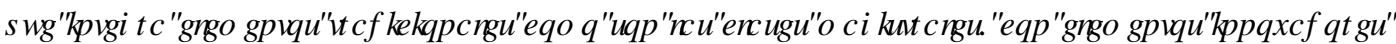

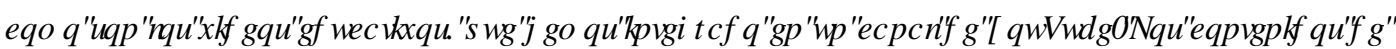

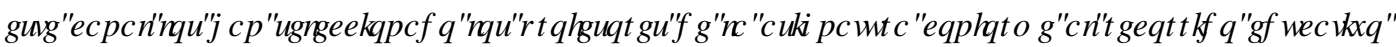

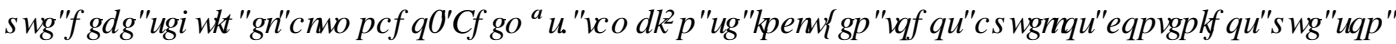
SLRSXHMRVISRUHOHWKGDQMDAR

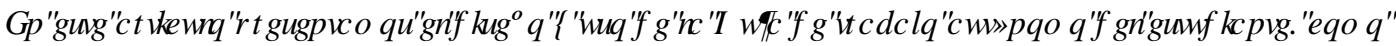

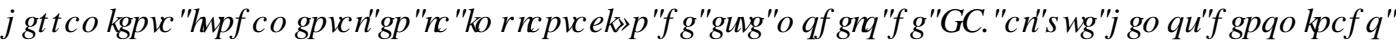

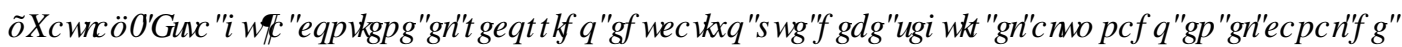

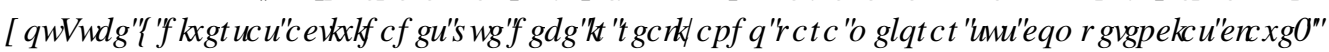

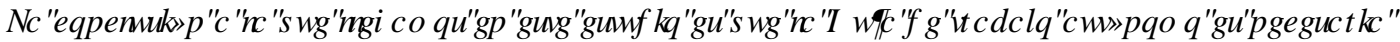

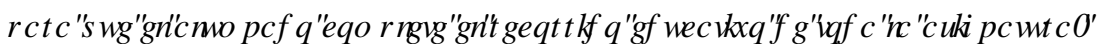

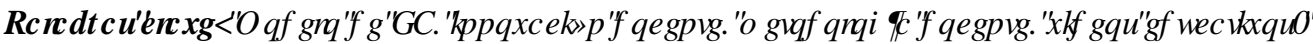




\section{Introducción}

Esta investigación comenzó con la premisa fundamental de ayudar a nuestro alumnado a mejorar su aprendizaje y lograr todos los objetivos educativos en las asignaturas, cuya temática está relacionada con el Cálculo Numérico y que se imparten en la Universidad de Jaén.

En una primera fase, diseñamos un modelo mixto de enseñanza-aprendizaje, en el que se compaginan elementos docentes tradicionales, como son las clases magistrales, con elementos innovadores, como son los videos educativos, al que hemos denominado "Vaula". (Huertas, Molina, Rosales, 2020).

El deseo de incorporar un nuevo modelo en nuestra metodología docente vino promovido por dos motivos fundamentales:

- El primero, porque el estudiantado acude a YouTube de forma habitual y reiterada para aprender lo que ya tenían que saber o para consolidar los conocimientos impartidos en el aula y que no les da tiempo a adquirir, bien porque necesiten más tiempo para su interiorización o bien porque la relación contenidoscréditos, es a veces, estresante debido al poco tiempo asignado para la gran cantidad de contenido a impartir. Estos contenidos los buscan y adquieren sin una guía, sin tener la seguridad de si son adecuados o no y si realmente son útiles en su aprendizaje.

- En segundo lugar, porque según afirman Castillo \& Carrillo (2012) "La adquisición de conocimientos sobre una materia no se realiza sólo en el tiempo en el que el alumno está en el aula", sobre todo si tenemos en cuenta que, como ya hemos comentado, este tiempo es bastante breve debido al ritmo tan arduo de los programas de algunas asignaturas. Por ello, es importante diseñar herramientas multimedia que den soporte al aprendizaje que se realiza fuera del aula.

Nuestro modelo de EA "Vaula", consta de 3 tipos de actividades:

1.- Clases Magistrales.

2.- Actividades asíncronas online que el alumnado realiza de forma autónoma, con una guía del recorrido educativo que debe seguir en el visionado de los vídeos. También entran dentro de estas actividades, los formularios online de autoevaluación, a fin de que el estudiante, tome conciencia de lo que ha aprendido, y revisión y búsqueda de material bibliográfico.

3.- Actividades síncronas online, como son las tutorías de forma que, si cualquier estudiante está trabajando los contenidos y tiene dudas, puede conectarse a la sala de tutoría y preguntar en ese momento.

Para llevar a cabo estas actividades hemos diseñado y creado 3 herramientas, que son la Guía de trabajo autónomo del estudiante, el Canal de YouTube "Cálculo Numérico para Ingenieros" y la Página Web "Algorítmica Numérica".

En este artículo mostraremos el diseño y uso de la guía de trabajo autónomo del estudiante, como herramienta fundamental en el modelo de EA "Vaula" y la posibilidad de que sea extrapolable a otras asignaturas del área de Matemática Aplicada.

El uso de las guías de trabajo autónomo surgió debido al cambio experimentado en la Enseñanza Superior, a raíz de la incorporación de la misma, al Espacio Europeo de Educación Superior. En ese momento y antes de iniciar el recorrido experimental de uso de esta guía, se trabajaba en guías de trabajo autónomo que estaban basadas en el concepto de que el alumno, no iba a aprender para superar su evaluación, sino que lo haría para su propia formación profesional (Gámiz et al. 2008). 


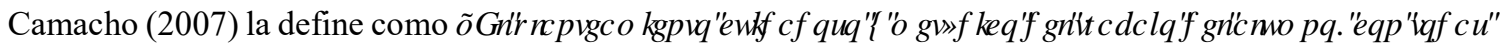

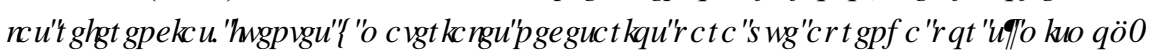

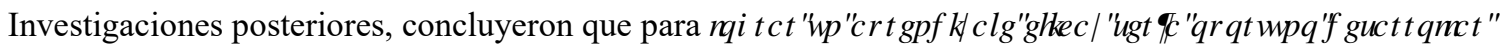

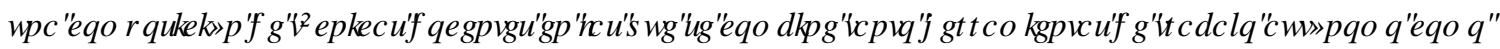

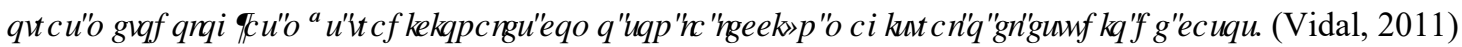

La guía de trabajo autónomo, para el modelo de EA "Vaula", la presentamos como un esquema de trabajo que ayuda al estudiante a organizar las tareas de modo secuencial (Saénz 1994,355)

El modelo se ha aplicado al bloque de Cálculo Numérico de las asignaturas y grados que aparecen en la tabla 1 y que se imparten en el Escuela Politécnica Superior de Jaén.

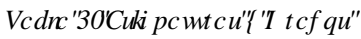

\begin{tabular}{cc}
\hline Asignatura & Grado \\
\hline Análisis y Métodos Numéricos & Grado en Ingeniería Informática \\
Matemáticas II & Grado en Ingeniería de Organización Industrial \\
Matemáticas II & Grado en Ingeniería Mecánica \\
Matemáticas II & Grado en Ingeniería Eléctrica \\
Matemáticas II & Doble grado en Ingeniería Eléctrica e Ingeniería Mecánica \\
Matemáticas II & Doble grado en Ingeniería Eléctrica e Ingeniería Electrónica \\
& Industrial \\
Matemáticas II & Doble grado en Ingeniería Mecánica e Ingeniería de Organización \\
Matemáticas II & Doble grado en Ingeniería Mecánica e Ingeniería Electrónica \\
& Industrial \\
\hline
\end{tabular}

La investigación que estamos realizando se enmarca en el Proyecto de Innovación Docente "Elaboración de recursos audiovisuales para el progreso del aprendizaje en conocimientos básicos de Cálculo Numérico para el alumnado de la UJA" concedido por la Universidad de Jaén.

Esta investigación se hace sobre una muestra de 430 estudiantes distribuidos por sexo, edad y asignatura, según la tabla 2

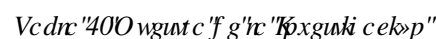

\begin{tabular}{ccccccc}
\hline Asignatura & \%Total & \%Mujeres & \%Hombres & \% $\leq \mathbf{2 0}$ & $\mathbf{\% 2 1 - 2 5}$ & \% $\geq \mathbf{2 5}$ \\
\hline $\begin{array}{c}\text { Análisis y Métodos } \\
\text { Numéricos }\end{array}$ & 47.2 & 14.8 & 85.2 & 78.9 & 17.2 & 3.9 \\
Matemáticas II & 52.8 & 22 & 78 & 83.7 & 13.2 & 3.1 \\
\hline
\end{tabular}




\section{Objetivos}

El objetivo general de este estudio es diseñar la Guía de trabajo autónomo del alumnado y estudiar el grado de exportabilidad que tiene, tanto el Modelo de EA, como la Guía, a otras asignaturas del área de Matemáticas.

Para lograr este objetivo, planteamos una serie de tareas a seguir:

- Realizar una planificación temporal de la materia de la asignatura.

- Seleccionar el contenido que se debe impartir en el aula mediante clase magistral, el contenido a debatir en el aula y el contenido que el alumnado puede visionar en video.

- Diseñar aquellas actividades que el alumnado debe realizar, de manera autónoma, para mejorar su aprendizaje de la materia y sus competencias.

- Establecer el contenido de la Guía de autoaprendizaje.

Al realizar estas tareas no debemos perder de vista los objetivos de aprendizaje que queremos para nuestros estudiantes. Estos son:

- Adquirir un aprendizaje significativo de la materia.

- Ser conscientes de ese grado de aprendizaje

- Desarrollar un amplio rango de actividades prácticas

- Adquirir competencias necesarias para su futuro profesional.

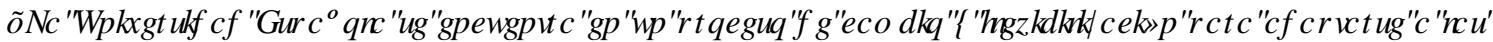

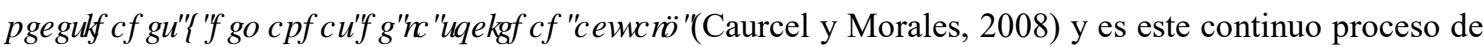
adaptación el que nos mueve a integrar material multimedia en nuestra metodología docente.

\section{Desarrollo de la Innovación}

Tras el diseño teórico del Modelo de EA "Vaula" llegó la fase de su implementación. Comenzamos ésta con el diseño y creación de las herramientas necesarias, para posteriormente, hacer un trabajo de investigación que nos permitiera conocer el grado de aceptación que tendrían nuestros estudiantes ante la inclusión de esta metodología en su modelo de aprendizaje.

Pudimos comprobar que el grado de aceptación es de un 96,7\%. El 3,3\% restante corresponde a no sabe/no contesta.

Puesto que el grado de aceptación fue muy alto, el siguiente paso fue diseñar y crear el canal de YouTube y dotarlo de contenido, para seguir trabajando con la muestra y conocer su valoración.

El diseño del canal se ha realizado en base a tres niveles de contenido. Un nivel básico dónde se trabajan los fundamentos matemáticos de distintos métodos numéricos. Un nivel intermedio, dónde se aplican esos fundamentos matemáticos a diversos problemas de la vida real y, por último, un nivel avanzado, dónde aplicamos los fundamentos matemáticos de resolución de métodos numéricos a problemas reales de la profesión de Ingeniería, en sus distintas ramas.

La respuesta del alumnado a la creación del canal ha sido positiva en el $100 \%$ de la muestra, obteniendo una valoración media de 9.6

Una vez enmarcados en este contexto comenzamos con la elaboración de la guía, para ello seguimos los siguientes pasos: 
a) Contenidos que vamos a tratar:

Tema 1: Resolución Numérica de Ecuaciones

Tema 2: Aproximación de funciones

Tema 3: Integración Numérica

Tema 4: Resolución Numérica de PVI

b) Distribución temporal de estos contenidos:

Las dos asignaturas en las que estamos realizando esta investigación son cuatrimestrales. Análisis y Métodos Numéricos se imparte en el primer cuatrimestre y Matemáticas II en el segundo. La primera tiene una asignación de créditos inferior al que tiene la segunda. Podemos ver esta distribución en las tablas 3 y 4 .

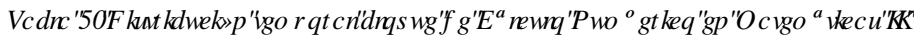

\begin{tabular}{cc}
\hline Asignatura & Grado \\
\hline Semanas $1,2,3,4$ & Resolución Numérica de Ecuaciones \\
Semanas $5,6,7,8$ & Aproximación de Funciones \\
Semanas $9,10,11,12$ & Integración Numérica \\
Semanas $12,13,14,15$ & Resolución Numérica de PVI \\
\hline
\end{tabular}

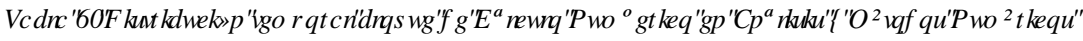

\begin{tabular}{cc}
\hline Asignatura & Grado \\
\hline Semana 12 & Resolución Numérica de Ecuaciones \\
Semana 13 & Aproximación de Funciones \\
Semana 14 & Integración Numérica \\
Semana 15 & Resolución Numérica de PVI \\
\hline
\end{tabular}

En la tabla 4, comprobamos que en esta asignatura el programa es muy agobiante y que la inclusión de elementos multimedia en la metodología didáctica puede resultar de gran ayuda para el alumnado.

c) Selección del contenido que se debe impartir en el aula mediante clase magistral, el contenido a debatir en el aula y el contenido que el alumnado puede visionar en video.

En clase magistral se impartirán los contenidos fundamentales de cada tema. Se debatirá en el aula los problemas más importantes y el estudiantado hará visionado de video de contenidos introductorios, que ya deben conocer y es importante que recuerden y repasen antes de tratar los contenidos fundamentales.

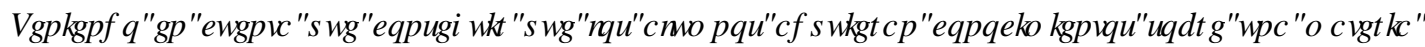

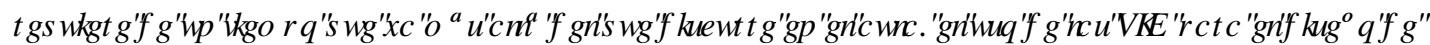

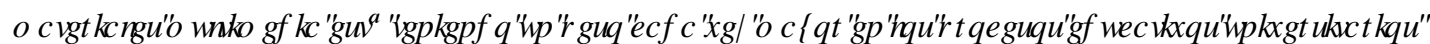

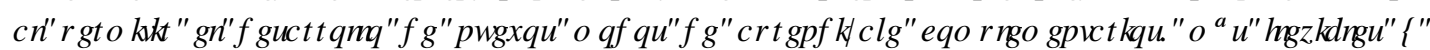


FRQMRCEAOMSRUHODOP QR. (Castillo\&Carrillo,2012). Creemos que esta distribución de contenido es la adecuada para motivar al estudiante y lograr mejorar y reforzar su aprendizaje.

d) Diseñar las actividades, entre las que se encuentran formularios de autoevaluación, problemas, exposiciones en clase y participación en foros de debate.

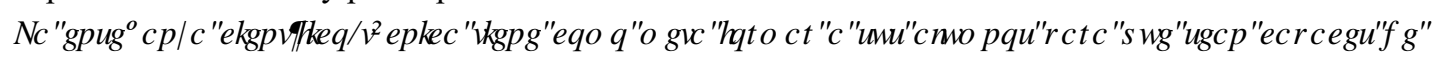

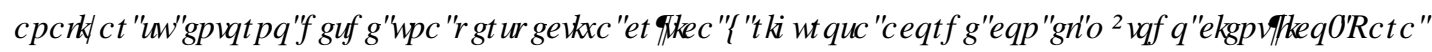

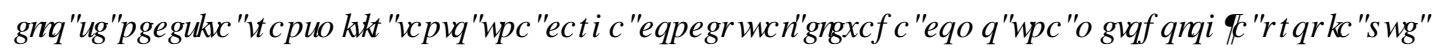

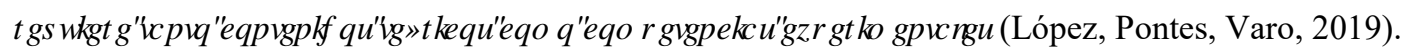

Es por ello que pretendemos que el estudiante tome conciencia de su grado de aprendizaje mediante cuestionarios de autoevaluación, para que sea él mismo quien decida en que área debe incidir y/o reforzar. Usamos la metodología de desarrollo de habilidades mediante la resolución de problemas, con lo que aprenderán técnicas y estrategias que serán de aplicación a los problemas tanto de la vida real como de su profesión. Por último, la exposición en clase y los debates fomentarán el aprendizaje pues "aprenden para enseñar".

e) Con todo lo expuesto se desarrolla el recorrido educativo que debe seguir cualquier estudiante en su autoaprendizaje, para cada una de las asignaturas expuestas.

Este recorrido se establece según asignatura, programa y créditos asignados al programa.

\section{Resultados}

El resultado de este estudio concluye con la creación de la Guía de autoaprendizaje que tiene la siguiente estructura:

1.- Introducción y objetivos.

2.- Conocimientos introductorios que el alumnado debe reforzar de cada tema, junto con la asignación temporal y semana del cuatrimestre en que debería trabajarlos. Deberá realizar un cuestionario de autoevaluación por cada tema.

3.- Actividades que debe realizar después de la exposición de los contenidos fundamentales en clase. El estudiante deberá realizar una relación de problemas del tema, que tendrá resueltos en el canal de YouTube. Con esta relación desarrollará las habilidades necesarias para poder, posteriormente, exponer en clase la solución de determinadas cuestiones que se le plantearán.

4.- Exposiciones que debe preparar para realizarlas en clase. El alumnado contará con una relación de cuestiones, relacionadas con el contenido que se esté tratando, que podrá preparar previamente para exponerlas posteriormente en clase.

5.- Tras cada una de estas exposiciones se realizará un debate, en el que se discutirá si la solución aportada es única, la mejor, etc.

Con ello pretendemos potenciar varios valores a la pedagogía de las ciencias, como son: promover un mejor rendimiento cognitivo, proporcionar una visión más amplia de las experiencias que permite relacionar los conceptos con el entorno más cercano y facilitar la recolección y presentación de datos empíricos (Webb, 2005)

Romero y Quesada (2014) también nos dicen que la incorporación de las TIC puede ayudar a resolver problemas cuantitativos, entender conceptos físicos o desarrollar destrezas espaciales, habilidades todas 
ellas necesarias para ejercer la profesión de ingeniería. Así mismo, recalcan la importancia de usar metodologías basadas en las TIC con la finalidad de fomentar la interacción del alumnado a través de debates, ya sean en el aula o en foros digitales.

\section{Conclusiones}

De este trabajo se desprende que la incorporación de las tecnologías de la información y comunicación a la enseñanza universitaria en el área de Matemática Aplicada, se convertirá en un recurso básico de la metodología educativa.

Gran parte de la enseñanza sigue siendo unidireccional, basada en información estática y, por tanto, la incorporación de un nuevo modelo de EA en el que el alumnado debe realizar una serie de actividades dirigidas y hacer un visionado de material multimedia, fomenta la participación de los estudiantes. Hecho este que los docentes siempre estamos buscando, por lo positivo que resulta para el aprendizaje.

Navaridas y Jiménez (2016) encuentran que los ambientes de aprendizaje considerados más eficaces por los estudiantes son el estudio personal y las explicaciones del profesor.

La Guía de trabajo autónomo, se diseña para que el estudiante la utilice en su estudio personal, para que rentabilice ese tiempo de estudio y realice actividades que le ayuden en su aprendizaje y en su desarrollo académico y, como ya hemos comentado en la introducción, la presentamos como un esquema de trabajo que ayuda al alumnado a organizar las tareas de modo secuencial (Saénz 1994,355).

\section{Referencias}

CABERO, J. \& MARÍN, V. (2014). "Posibilidades educativas de las redes sociales y el trabajo en grupo. Percepciones

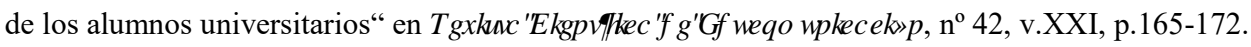

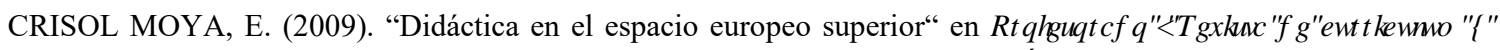
IRLP DFIYQ GHOSLRIHRURR, Vol.13, No 1, p. 387-394. Es reseña de : “ RODRÍGUEZ FUENTES, A., CAURCEL CARA, M.J. y RAMOS GARCÍA, A.M.(Coords.) (2008). "Didáctica en el espacio europeo superior “.

CRISOL MOYA, E. \& ROMERO LÓPEZ, M.A. (2013). "Las guías de trabajo autónomo a través de Moodle. Opinión de los estudiantes. Una experiencia en la Universidad de Granada" en 8 \&6. Vol.12, No. 23, enero-julio,2013. P. 159175

GÁMIZ, V., RODRÍGUEZ, M. J. y ROMERO, M. A. (2008). "Las herramientas didácticas en la universidad" en A. Rodríguez, M.J. Caurcel y A. M. Ramos (Coords), ' IG FUFDHQHOQ USDFIRU( XLRSHRIGH( GXFDFIy Q6XSHURU]* XtDVGH WDEDNFDXWYRP R. Madrid: EOS Universitaria, p. 71-92.

GARCÍA, A. y RODRÍGUEZ, A. (2008). "Las guías de trabajo autónomo en la universidad" en A. Rodríguez, M.J. Caurcel y A.M. Ramos (Coords.), ' IG FUFD HQ HD( ISDFIRD ( XURSHR GH ( GXFDFIY Q 6XSHURUT * XTDV GH WDEDWI DXWYQRP R. Madrid: EOS Universitaria, p. 93-117.

GÓMEZ, M., ROSES, S. y FARIAS, P. (2012). "El uso académico de las redes sociales" en 5HIWD\&IHQMIFDGH ( GXFRP XQFDFFY $Q \mathrm{n}^{\circ} 38$, v. XIX, p. 131-138.

GUTIÉRREZ PORLÁN, I. (2011). "Aprendizaje con redes sociales : Tejidos educativos para los nuevos entornos" en

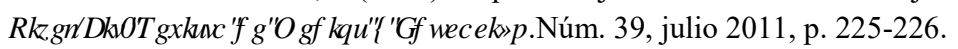

HUERTAS, A., MOLINA, M.F. y ROSALES, M.C. (2020). "La enseñanza universitaria mediante la combinación de

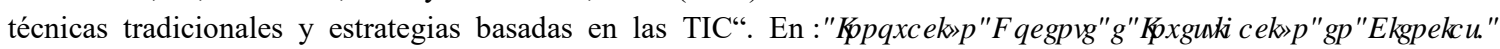

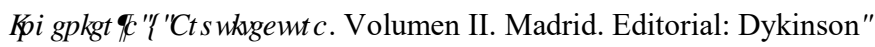


JIMÉNEZ CASTILLO, D. y MARÍN CARRILLO, G.M. (2012). “Asimilación de contenidos y aprendizaje mediante el uso de videotutoriales” en ( QMKDQJD प7HDFKQJ, 30, 2-2012, p.63-79.

LÓPEZ-QUINTERO, J. L., PONTES-PEDRAJAS, A. y VARO-MARTÍNEZ, M. (2019ロШ'Las TIC en la enseñanza científico-técnica hispanoamericana : Una revisión bibliográfica“. ' IJLDOC GXFDURQ5 HIIHZ-Number 35 June 2019-

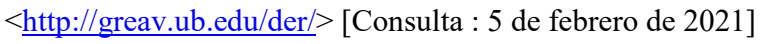

LOZANO DÍAZ, A., GONZÁLEZ MORENO, M.J. y CUENCA PIQUERAS, C. (2020). "Youtube comorecurso

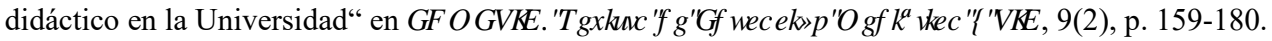

NAVARIDAS, NALDA, F., JIMÉNEZ TRENS, M. A. y FERNÁNDEZ ORTÍZ, R. (2016). "El aprendizaje de competencias en la Universidad : expectativas predictivas y niveles de confirmación de los estudiantes" en Revista Española de Pedagogía. No 264, mayo-agosto 2016, p. 337-356.

RODRÍGUEZ FUENTES, A., CAURCEL CARA, M.J. y RAMOS GARCÍA, A.M.(Coords.) (2008). ' IG FWFDHQHD HSDFIRIHURSHRIXSHURU Madrid : Editorial EOS.

ROMERO ARIZA, M. QUESADA ARMENTEROS, A. (2014). "Nuevas tecnologías y aprendizaje significativo de las ciencias" en ( QMHEDJDGHDQK\&IHQFID, Núm.32.1, p.101-115.

ROMERO, M. A. \& CRISOL, E. (2009). "La guía de trabajo autónomo en la experiencia ECTS de la Titulación de

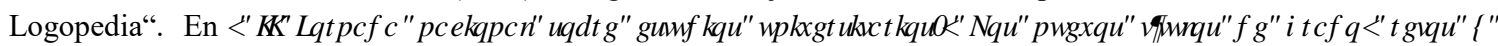
RSRLXXGODOH. Castellón. Edita : Universitat Jaume I. Servei de Comunicació i Publicacions. Castellón. 125-126.

ROMERO LÓPEZ, M. A. \& CRISOL MOYA, E. (2012). "Las guías de aprendizaje autónomo como herramienta didáctica de apoyo a la docencia“ en ( VXHDD\$ EIHWD, 15, p. 9-31.

SÁENZ BARRIO, O. (1994). "Métodos autodirectivos e individualizados“en Didáctica general : un enfoque curricular. Coord. Óscar Sáenz Barrio, p. 341-380.

VIDAL SALAZAR, M. D., FERRÓN VÍLCHEZ, V. y DE LA TORRE RUIZ, J.M. (2011). “La metodología del aprendizaje por indagación en la enseñanza universitaria : ejemplos concretos de aplicación“. Póster. En \&RQJHMR ,QUACDFIRQDOCH, QQRYDFY Q' RFHQUH Cartagena. Cartagena : Universidad Politécnica de Cartagena, p.75

WEBB, M. E. (2011). "Affordances of ICT in science learning: implications for an integrated pedagogy" en ,QMHQDURQDO-RXLQDORI6FHQFH( GXFDMRQ 27:6, p.705-735. 